\title{
Spin-to-Orbital Angular Momentum Mapping of Polychromatic Light
}

\author{
Mushegh Rafayelyan and Etienne Brasselet \\ Univ. Bordeaux, CNRS, LOMA, UMR 5798, F-33400 Talence, France
}

(Received 4 July 2017; published 25 May 2018)

\begin{abstract}
Reflective geometric phase flat optics made from chiral anisotropic media recently unveiled a promising route towards polychromatic beam shaping. However, these broadband benefits are strongly mitigated by the fact that flipping the incident helicity does not ensure geometric phase reversal. Here we overcome this fundamental limitation by a simple and robust add-on whose advantages are emphasized in the context of spin-to-orbital angular momentum mapping.
\end{abstract}

DOI: 10.1103/PhysRevLett.120.213903

Since the invention of thin lenses by Fresnel in the 1820s in the framework of lighthouse optics [1], tremendous development of flat optics has taken place. Nowadays, the design of subwavelength-thin optical elements enabling almost arbitrary intensity, phase, and polarization transformations is well established and nanofabrication tools made possible their realization [2-4]. In this context, the development of geometric phase optical elements has aroused great interest. Indeed, two decades after the proposal by Bhandari [5], which was followed by first experimental realizations in the early 2000s [6,7], now such optical elements are products of the photonics industry and the underlying physics relying on spin-orbit interactions of light [8] is well understood.

The principle of such devices is grasped by considering a transparent slab of uniaxial medium with space-variant inplane optical axis orientation angle $\psi(x, y)$ and uniform half-wave birefringent phase retardation. As shown by Bhandari [5], an incident circularly polarized plane wave propagating along the $z$ axis emerges from the sample with the orthogonal circular polarization state and additional geometric phase factor proportional to $\sigma \psi(x, y)$ where $\sigma= \pm 1$ is the helicity of the incident field. The geometrical information $\psi(x, y)$ can thus be transferred to the wave front of the output light field and reversed on demand by flipping the incident helicity $(\sigma \rightarrow-\sigma)$. Since the half-wave retardation condition is satisfied for a specific wavelength, these geometric phase optical elements are monochromatic by design.

A few designs have been proposed to achieve achromatic transmissive [9-11] or reflective [12] geometric phase shaping though being restricted to $4 \pi$-helical phase bulky shapers with poor on-axis spatial resolution. Still, highresolution transmissive flat optics with enhanced polychromatic behavior can be achieved by introducing a multitwisted anisotropic structure [13]. Recently, a simpler option has been proposed [14], which combines the twisted nature of chiral liquid crystals (i.e., cholesterics) that may lead to circular Bragg reflection [15] with well-developed liquid crystal surface patterning technology [16,17]. The geometric (Berry) phase nature of such (Bragg) reflective optical elements made of chiral and anisotropic media has been revealed independently in Refs. [18,19]. In particular, polychromatic optical vortex beam generation using "Bragg-Berry" mirrors [20-24] unveiled a promising option. However, as previously noticed in Ref. [20], flipping the incident helicity does not ensure geometric phase reversal, which is a major drawback. Here we report on a simple and robust way to solve this problem with altering neither the polychromaticity nor the rotational invariance, in contrast to what happens at large incidence angle [20-22]. This is done by using a rear mirror [25], see Fig. 1(a), which displays a cholesteric slab sandwiched between a glass substrate (at $z=0$ ) and a standard mirror (at $z=L$ ) that are associated with boundary layers providing identical in-plane liquid crystal orientation patterns given by the spatially varying angle $\psi_{\text {surf }}(x, y)$. The demonstration is made in the context of spin-to-orbital angular momentum mapping, which covers various aspects of classical and quantum optics [26], and for which the polychromatic option is not available to date.

Cholesterics are characterized by a helical structure with pitch $p$ that is the distance over which the local liquid crystal optical axis rotates by $2 \pi$ around the $z$ axis and by handedness $\chi= \pm 1$; see Fig. 1(b). From the optics of uniform cholesterics [i.e., $\psi_{\text {surf }}(x, y)=\psi_{0}$ with $\psi_{0}$ constant], light propagating along the helix axis experiences helicity-preserved Bragg reflection for the incident helicity $\sigma=-\chi$. This is associated with a photonic band gap defined by $p n_{\perp}<\lambda<p n_{\|}, n_{\|, \perp}$ being the refractive index along and perpendicular to the liquid crystal director $[29,30]$. On the other hand, light with $\sigma=+\chi$ is transmitted through the cholesteric. In our case, such light is reflected by the rear mirror, which flips the helicity. Backward light is thus Bragg reflected without helicity change by the back side of the cholesteric. Ensuing helicity-flipped reflection from the rear mirror is eventually transmitted through the cholesteric at fixed helicity. The 


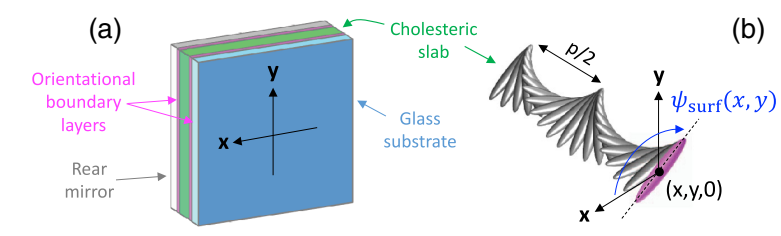

(c) Bragg reflected light

(d) Non-Bragg reflected light

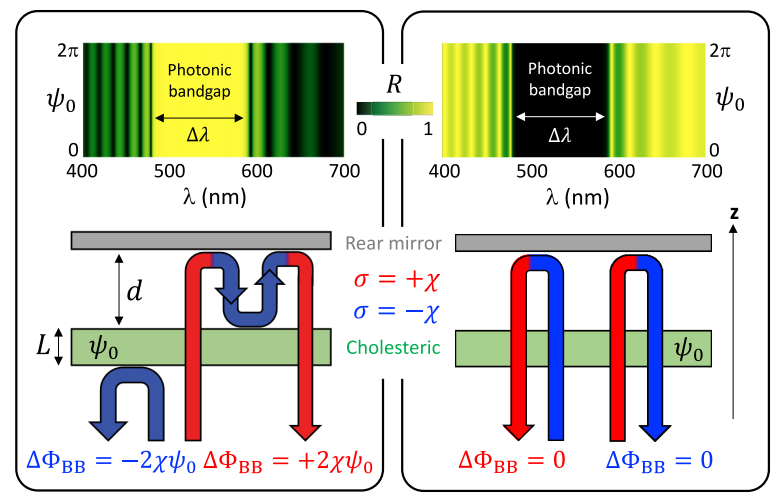

FIG. 1. (a) Generic mirror-backed Bragg-Berry optical element. (b) Helical cholesteric ordering for $\chi=+1$. (c) Calculated helicity-independent reflectance spectra and principle of the helicity-controlled geometric phase for a uniform surface alignment $\psi_{\text {surf }}=\psi_{0}$, for Bragg reflected light. (d) Same as in panel (c) for non-Bragg reflected light. Simulations are made using $4 \times 4$ Berreman formalism [27,28] at normal incidence, with $n_{\text {glass }}=1.52, n_{\|}=1.72, n_{\perp}=1.42, p=340 \mathrm{~nm}, \chi=-1, L=10 p$, $d=0$ and assuming a perfect mirror.

process is sketched in Fig. 1(c) and discussed in Ref. [31]. Arbitrarily polarized light is thus fully Bragg reflected for optical frequencies inside the band gap; see Fig. 1(c). Outside the band gap, only a fraction of the incident light is Bragg reflected, see Fig. 1(c), while the rest is reflected by the structure with flipped helicity, as depicted in Fig. 1(d).

The geometric Berry phase of Bragg reflected light, $\Phi_{\mathrm{BB}}$, strongly depends on the incident helicity, wavelength, and cholesteric orientational boundary conditions, see Fig. 2. However, the differential geometric phase $\Delta \Phi_{\mathrm{BB}}=$ $\Phi_{\mathrm{BB}}\left(\psi_{0}\right)-\Phi_{\mathrm{BB}}(0)$ does not depend on the wavelength. Namely, $\Delta \Phi_{\mathrm{BB}}=2 \sigma \psi_{0}$, and the generalization to spacevariant boundary conditions gives $\Delta \Phi_{\mathrm{BB}}=2 \sigma \psi_{\text {surf }}(x, y)$, which is the main result of this work.

Experimentally, this is investigated by preparing a $10 \mu$ m-thick Bragg-Berry optical element made of the chiral liquid crystal material SLC79 (BEAM Co). The chiral film is sandwiched between two glass substrates coated by an azobenzene-based surface-alignment layer providing a $q$-plate design for the orientational boundary conditions [32]. Namely, $\psi_{\text {surf }}=q \varphi$, with $q=1$ and $\varphi=\arctan (y / x)$. The cholesteric pitch is adjusted to provide a Bragg reflection spectrum centered around $\lambda \gtrsim 530 \mathrm{~nm}$ and the photonic band gap width is measured to be $\simeq 75 \mathrm{~nm}$. Without the back mirror add-on, this optical element behaves as a polychromatic Bragg-Berry vortex generator (BBVG) for $\sigma=-\chi$ only, hence loosing all the

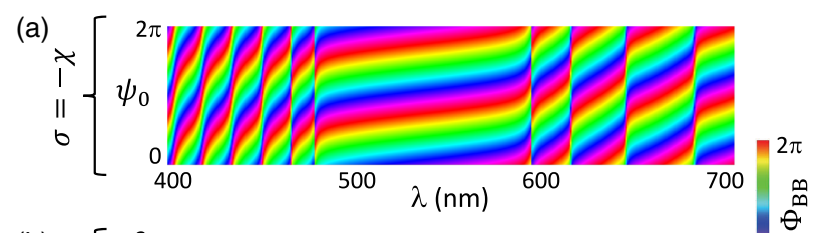

(b)

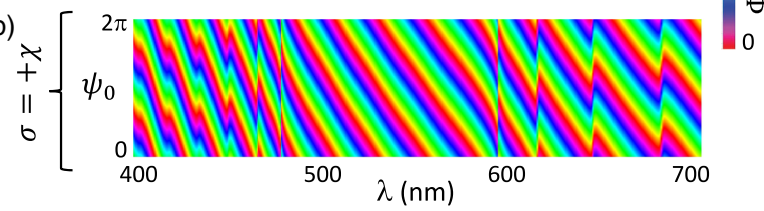

FIG. 2. Bragg reflected geometric phase spectra for the same parameters as in Fig. 1 for incident helicity $\sigma=\mp \chi$ (a), (b).

assets of a $q$ plate [26,32], as shown in the left part of Fig. 3. In this figure, the direct observation of the reflected intensity profile under incident helicity $\sigma= \pm \chi$ and various spectral cases are reported. In contrast, the latter issue is solved in the presence of the back mirror, as shown in the right part of Fig. 3. This supports the creation of a BraggBerry $q$ plate (BBQP) allowing broadband spin-to-orbital angular momentum mapping.

First, we recall the useful formalism of high-order Poincaré spheres of integer order $\ell$ [33], $\mathcal{P}_{\ell}$; see Fig. 4. Each point on the sphere corresponds to a unique optical state $\boldsymbol{\Psi}_{\ell}$ having two degrees of freedom,

$$
\Psi_{\ell}(a, b)=a e^{-i \ell \varphi} \mathbf{c}_{+}+b e^{+i \ell \varphi} \mathbf{c}_{-}
$$

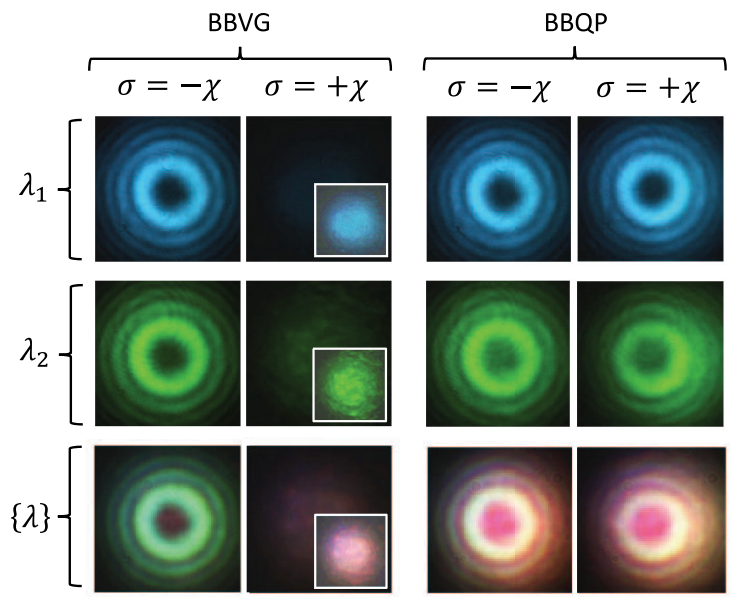

FIG. 3. Experimental comparison of the helicity-dependent vortex beam generation between BBVG and BBQP $(\chi=-1$, $q=1)$. Images refer to the reflected beam intensity at nearnormal incidence $\left(1^{\circ}\right.$ incidence angle) collected at $\sim 1 \mathrm{~m}$ from the element illuminated by a circularly polarized collimated supercontinuum laser beam with helicity $\sigma$, diameter $\sim 1 \mathrm{~mm}$, with interference filters centered on $\lambda_{1}=500$ and $\lambda_{2}=550 \mathrm{~nm}$ with $10 \mathrm{~nm}$ full width at half maximum transmission spectrum, or not $(\{\lambda\})$. For each pair of panels, the images are recorded at fixed acquisition time for the camera. Insets refer to enhanced-signal images to stress the absence of geometric phase shaping of the residual Fresnel reflection from the BBVG glass substrate. 


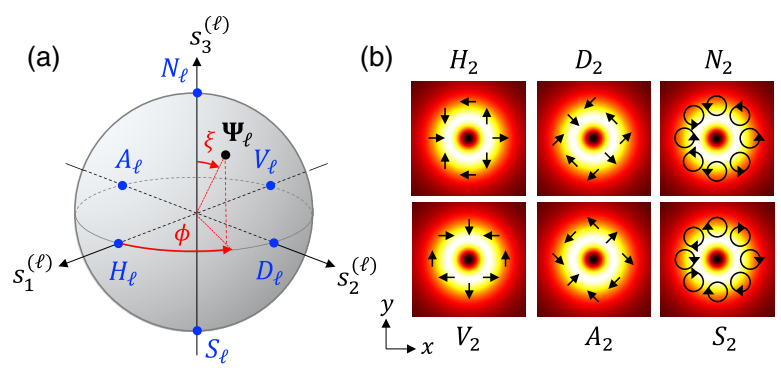

FIG. 4. (a) High-order Poincaré sphere $\mathcal{P}_{\ell}$ where any state $\Psi_{\ell}(a, b)$ given by Eq. (1) corresponds to a point parametrized by the angles $(\xi, \phi)$ with $\tan (\xi / 2)=|b / a|$ and $\phi=\arg (a / b)$. Six particular states are indicated and depicted in panel (b), where farfield intensity and polarization patterns are shown assuming an incident Gaussian beam. $N_{\ell}$ and $S_{\ell}$, respectively, refer to circularly polarized vortex fields associated with $s_{z}= \pm 1$ and $l_{z}=\mp 1 ; H_{\ell}, D_{\ell}, V_{\ell}$ and $A_{\ell}$, respectively, refer to space-variant linearly polarized beams having azimuth angle of the form $\ell \varphi$, $\ell \varphi+\pi / 4, \ell \varphi+\pi / 2$ and $\ell \varphi+3 \pi / 4$.

where $(a, b)$ are complex numbers, $|a|^{2}+|b|^{2}=1$, and $\mathbf{c}_{ \pm}=(\mathbf{x} \pm i \mathbf{y}) / \sqrt{2}$ refers to the circular polarization unit vectors in the Cartesian unit basis $(\mathbf{x}, \mathbf{y}, \mathbf{z})$. The mapping of the Poincaré sphere of polarization to high-order Poincaré sphere, namely, $\mathcal{P}_{0} \rightarrow \mathcal{P}_{\ell}$, is actually achieved inside the photonic band gap of a BBQP with handedness $\chi$ of order $q$ according to the unitary transformation

$$
\boldsymbol{\Psi}_{0}(a, b) \underset{\chi, q}{\stackrel{\mathrm{BBQP}}{\longrightarrow}} \Psi_{2 q}\left(b e^{i \Phi_{1}}, a e^{i \Phi_{2}}\right),
$$

where constants $\Phi_{1,2}$ depend on $\lambda$, material properties and considered geometry. Isomorphisms between $\phi$ and $\Phi_{1,2}$ ensure the full mapping between $\mathcal{P}_{0}$ and $\mathcal{P}_{2 q}$.

Without lack of generality, experiments are made using a collimated Gaussian laser beam with $\lambda=532 \mathrm{~nm}$ and diameter $\sim 1 \mathrm{~mm}$ following the setup of Fig. 5. The reflected beam is analyzed by direct polarimetry (i.e., without reference beam) and by interferential measurements (i.e., with coaxial reference beam). The predicted mapping given by Eq. (2) is illustrated by analyzing the reflected far field for a representative set of incident states, namely, $\mathbf{\Psi}_{0}^{(i)}=\left(S_{0}, N_{0}, H_{0}, D_{0}, V_{0}, A_{0}\right)$. The case of incident circularly polarized light is summarized in Fig. 6 where the top or bottom rows refer to $\boldsymbol{\Psi}_{0}^{(i)}=\left(S_{0}, N_{0}\right)$. The observed doughnut intensity profile is the result of on-axis optical phase singularity. Maximum [Figs. 6(c) and 6(h)] and minimum [Figs. 6(d) and 6(g)] visibility of the interference pattern, respectively, implies parallel and orthogonal circularly polarized reflected and reference fields. On the other hand, double-arm spirals reveal phase singularities with topological charge \pm 2 , whose sign is inferred from the spiral handedness. This demonstrates the transformations $S_{0}^{(i)} \rightarrow N_{2}^{(r)}$ and $N_{0}^{(i)} \rightarrow S_{2}^{(r)}$. Similar analysis is performed for an incident linear polarization,

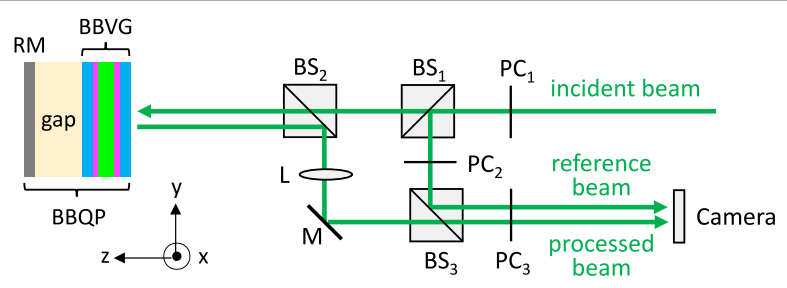

FIG. 5. Sketch of the experimental setup. Here the gap between rear mirror (RM) and BBVG corresponds to an actual experimental situation. mirror $(M)$; beam splitters $\left(\mathrm{BS}_{1,2,3}\right)$; lens with focal length $f=0.5 \mathrm{~m}$ in $f-f$ configuration with respect to the $\mathrm{BBQP}$ and the camera $(L)$; polarizer $(P)$; polarization controllers $\left(\mathrm{PC}_{1,2,3}\right)$.

$\Psi_{0}^{(i)}=H_{0}$, which is reported in the top row of Fig. 7. In that case, the reflected field is a superposition of contracircularly polarized optical vortex beams with opposite topological charges two and equal weights; see Figs. 7(c) and 7(d). This agrees with the expected transformation $H_{0}^{(i)} \rightarrow \Psi_{0}^{(r)}\left(e^{i \Phi_{1}} / \sqrt{2}, e^{i \Phi_{2}} / \sqrt{2}\right)$. More details are obtained from spatially resolved polarimetric analysis of the reflected field (see Supplemental Material [25]) that unveils an inhomogeneous linearly polarized beam carrying polarization singularity with topological charge 2, see Fig. 7(e), and average degree of linear polarization equals to 0.87 . More generally, the transformations $X_{0}^{(i)} \rightarrow X_{2}^{(r)}$ with $X=$ $(H, D, V, A)$ are thus validated. Note that this implies a tuning of the gap (see Fig. 5) to ensure $\Phi_{1}-\Phi_{2}=0$.

The preceding remark suggests that the isotropic phase delay $\Xi_{\text {iso }}=8 \pi d / \lambda$ associated with the optical path $4 d$ of the reflected field component with helicity $\sigma=\chi$, see Fig. 1(c), enables control of the BBQP at fixed incident polarization. Indeed, a dephasing $\Xi_{\text {iso }}$ between the two contracircularly polarized components corresponds to an angular displacement by an angle $\Delta \phi=\Xi_{\text {iso }}$ along a line of latitude on $\mathcal{P}_{2 q}$. This is implemented by placing the rear

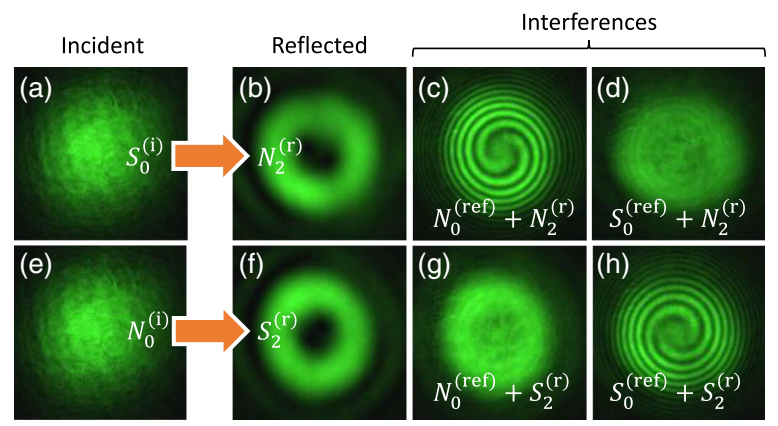

FIG. 6. Analysis of the reflected far field for $\Psi_{0}^{(i)}=S_{0}$ (top row) and $\Psi_{0}^{(i)}=N_{0}$ (bottom row). Interference intensity patterns are obtained by coaxial coherent superposition with circularly polarized reference beam with helicity \pm 1 , namely $\Psi_{0}^{(\text {ref })}=\left(N_{0}, S_{0}\right)$. Superscripts $(i),(r)$, and (ref) refer to incident, reflected, and reference fields. 


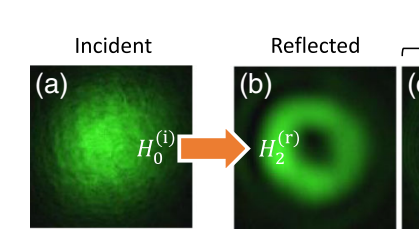

Interferences

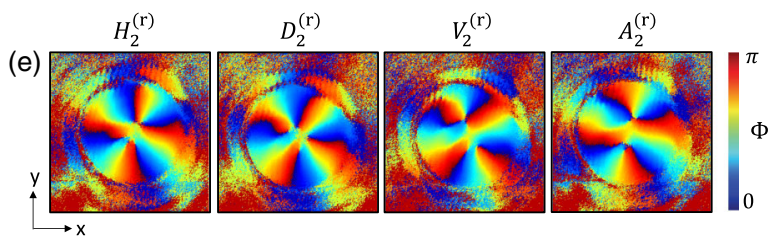

FIG. 7. Top row: Analysis of the reflected far field for $\Psi_{0}^{(i)}=$ $H_{0}$ with a presentation similar to that of Fig. 6. Bottom row: Reconstruction of the polarization azimuth angle $(\Phi)$ of the reflected far field from spatially resolved Stokes polarimetry for the four distinct cases leading to $H_{2}^{(r)}, D_{2}^{(r)}, V_{2}^{(r)}$, and $A_{2}^{(r)}$.

mirror on a piezoelectric stage. The experiment is carried out by using a linearly polarized incident beam, which corresponds to a revolving exploration of the equator of $\mathcal{P}_{2 q}$, see Fig. 8. The rotating four-lobe intensity pattern with a period that corresponds to $\Delta \Xi_{\text {iso }}=8 \pi$ agrees with the expected $2 \pi$ rotation of a $4|q|$-lobe intensity pattern for $\Delta \Xi_{\text {iso }}=8|q| \pi$ in the general case.

Reconfiguration of the BBQP at fixed incident polarization is also possible by imparting a birefringent phase retardation $\Xi_{\text {aniso }}$ to the back-reflected light. This allows us to turn on and off the geometric phase shaping of the incident light with $\sigma=\chi$ by switching $\Xi_{\text {aniso }}$ from 0 to $\pi / 2$. Indeed, $\Xi_{\text {aniso }}=\pi / 2$ acts as a quarter-wave plate and its combination with the rear mirror behaves as an effective helicity-preserving reflector, as sketched in Figs. 9(a) and 9(b). Consequently, one can switch from high-order Poincaré sphere $\mathcal{P}_{\ell}$ to hybrid-order Poincaré sphere [34] $\mathcal{P}_{\ell, 0}$ with $\mathcal{P}_{\ell, \ell^{\prime}}$ defining the optical states

$$
\boldsymbol{\Psi}_{\ell, \ell^{\prime}}(a, b)=a e^{-i \ell \varphi} \mathbf{c}_{+}+b e^{+i \ell^{\prime} \varphi} \mathbf{c}_{-},
$$

and noting that $\mathcal{P}_{\ell, \ell} \equiv \mathcal{P}_{\ell}$. When $\ell \neq \ell^{\prime}$ one obtains socalled full Poincaré beams [35] that refers to inhomogeneously polarized beam whose local polarization states fully cover $\mathcal{P}_{0}$. Remarkably, our approach does not require either to prepare an incident field with non-zero orbital state or detuning the geometric phase optical element, in contrast to the case of usual $q$ plates. In our case, one can switch (a)

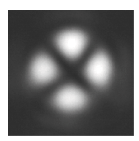

(b)

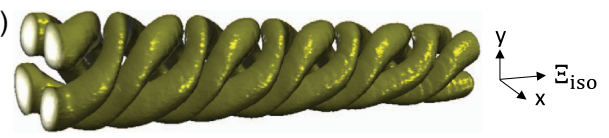

FIG. 8. Revolving exploration of the equator of $\mathcal{P}_{2 q}$ by isotropic phase control for $q=1$. Right part: Volumetric representation of the intensity patterns (without reference beam) obtained by setting $\mathrm{PC}_{1,3}$ as a linear polarizer, as $\Xi_{\text {iso }}$ varies over $\simeq 22 \pi$. Left part: transverse intensity profile snapshot.
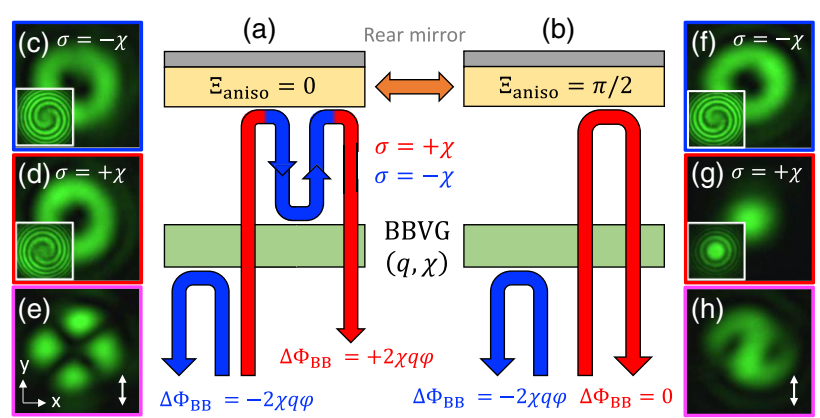

FIG. 9. (a), (b) On-demand activation of the geometric phase beam shaping of the incident light with $\sigma=\chi$ by tuning the birefringent phase delay $\Xi_{\text {aniso. }}$. Far field intensity profiles of the reflected light for incident helicity $\sigma= \pm \chi$ are shown in panels (c),(d) and (f),(g). Insets: interference patterns. The case of incident linear polarization is shown in panels (e) and (h), when $\mathrm{PC}_{3}$ is set as a linear polarizer $(\uparrow)$. Left or right panels refer to $\Xi_{\text {aniso }}=(0, \pi / 2)$, for $q=1$ and $\chi=-1$.

between vectorial vortex beams $\left(\boldsymbol{\Psi}_{\ell}\right)$ and full Poincaré beams of various kinds $\left(\boldsymbol{\Psi}_{\ell, 0}\right.$ or $\boldsymbol{\Psi}_{0, \ell}$ depending on $\left.\chi\right)$. This is illustrated in Figs. 9(c)-9(e) $\left(\Xi_{\text {aniso }}=0\right)$ and Figs. 9(f)-9(h) $\left(\Xi_{\text {aniso }}=\pi / 2\right)$.

As emphasized by Eq. (2), the dispersion on the highorder Poincaré sphere prevents to have spatially superimposed space-variant polarization patterns (except for $q=1 / 2$ ) although axisymmetric intensity distribution and topological phase shaping is properly achieved for each wavelength. Indeed, except for the north and south pole, any initial polychromatic state is mapped to a line of latitude on the output high-order Poincare sphere, each point corresponding to one wavelength. This could nevertheless be corrected in a satisfying manner by adding a dispersion compensation scheme, for instance, by inserting a slab with negative refractive index $\bar{n}=-\left[\left(n_{\perp}^{2}+n_{\|}^{2}\right) / 2\right]^{1 / 2}$ and thickness $\bar{L}=L / 2$ placed in between the back-mirror
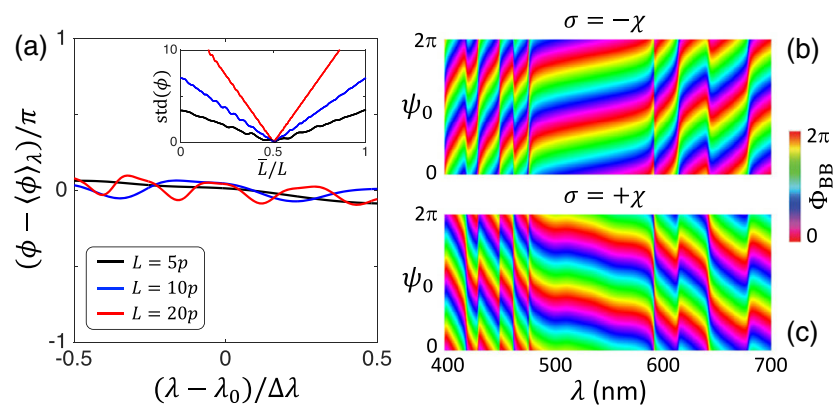

FIG. 10. (a) Dispersion of the reduced latitude angle, $\left(\phi-\langle\phi\rangle_{\lambda}\right) / \pi$ on the high-order Poincaré sphere for any incident polarization state for $\bar{L}=L / 2$ with $L=5 p, 10 p$ and $20 p$; $\lambda_{0}=p\left(n_{\|}+n_{\perp}\right) / 2$ refers to the central wavelength of the photonic band gap and $\Delta \lambda=p\left(n_{\|}-n_{\perp}\right)$ refers to the band gap width. Inset: standard deviation of $\phi(\lambda)$ in the photonic band gap versus the thickness $\bar{L}$ of the compensating layer. (b), (c) Bragg reflected geometric phase spectra for $L=10 p$ and incident helicities $\sigma= \pm \chi$. Material parameters are those of Fig. 1. 
and the Bragg-Berry slab of thickness $L$. The quality of such a dispersion management is quantitatively addressed in Fig. 10(a) for three different values of $L$. The corresponding spectral dependence of the helicity-dependent reflected phase for the Bragg-reflected light is shown in Figs. 10(b) and 10(c), whose comparison with Fig. 2 illustrates well the sought-after dispersion compensation inside the photonic band gap. Another option-bulkier but easier to implement and that provides perfect compensation-consists of process incident photons with opposite helicity by two independent Bragg-Berry vortex generators with identical $q$ and opposite $\chi$ using a two-arm configuration with equal optical path, as sketched in the Supplemental Material, Fig. S4 [25].

In conclusion, we note that although the present work is carried out in the specific framework of spin to orbital angular momentum mapping using $q$-plate design, it applies to all possible kinds of wave fronts that are shaped by the geometric phase. These results should therefore be useful for the optics and photonics community at large, even when dealing at the single photon level.

This study has been carried out with financial support from the French National Research Agency (Project No. ANR-15-CE30-0018).

*etienne.brasselet@u-bordeaux.fr

[1] J. Elton, Int. J. Hist. Eng. Tech. 79, 183 (2013).

[2] Y. Zhao, X.-X. Liu, and A. Alu, J. Opt. 16, 123001 (2014).

[3] N. Yu and F. Capasso, Nat. Mater. 13, 139 (2014).

[4] P. Genevet and F. Capasso, Rep. Prog. Phys. 78, 024401 (2015).

[5] R. Bhandari, Phys. Rep. 281, 1 (1997).

[6] Z. Bomzon, G. Biener, V. Kleiner, and E. Hasman, Opt. Lett. 27, 1141 (2002).

[7] G. Biener, A. Niv, V. Kleiner, and E. Hasman, Opt. Lett. 27, 1875 (2002).

[8] K. Y. Bliokh, F. J. Rodriguez-Fortuno, F. Nori, and A. V. Zayats, Nat. Photonics 9, 796 (2015).

[9] T. Wakayama, K. Komaki, Y. Otani, and T. Yoshizawa, Opt. Express 20, 29260 (2012).

[10] T. Wakayama, O. G. Rodríguez-Herrera, J. S. Tyo, Y. Otani, M. Yonemura, and T. Yoshizawa, Opt. Express 22, 3306 (2014).

[11] F. Bouchard, H. Mand, M. Mirhosseini, E. Karimi, and R. W. Boyd, New J. Phys. 16, 123006 (2014).

[12] N. Radwell, R. D. Hawley, J. B. Götte, and S. FrankeArnold, Nat. Commun. 7, 10564 (2016).

[13] Y. Li, J. Kim, and M. J. Escuti, Proc. SPIE Int. Soc. Opt. Eng. 8274, 827415 (2012).

[14] J. Kobashi, H. Yoshida, and M. Ozaki, Nat. Photonics 10, 389 (2016).
[15] M. Faryad and A. Lakhtakia, Adv. Opt. Photonics 6, 225 (2014).

[16] J. Kim, Y. Li, M. N. Miskiewicz, C. Oh, M. W. Kudenov, and M. J. Escuti, Optica 2, 958 (2015).

[17] P. Chen, B.-Y. Wei, W. Ji, S.-J. Ge, W. Hu, F. Xu, V. Chigrinov, and Y.-Q. Lu, Photon. Res. 3, 133 (2015).

[18] M. Rafayelyan, G. Tkachenko, and E. Brasselet, Phys. Rev. Lett. 116, 253902 (2016).

[19] R. Barboza, U. Bortolozzo, M. G. Clerc, and S. Residori, Phys. Rev. Lett. 117, 053903 (2016).

[20] M. Rafayelyan and E. Brasselet, Opt. Lett. 41, 3972 (2016).

[21] J. Kobashi, H. Yoshida, and M. Ozaki, Phys. Rev. Lett. 116, 253903 (2016).

[22] M. Rafayelyan, G. Agez, and E. Brasselet, Phys. Rev. A 96, 043862 (2017).

[23] J. Kobashi, H. Yoshida, and M. Ozaki, Mol. Cryst. Liq. Cryst. 646, 116 (2017).

[24] P. Chen, L.-L. Ma, W. Duan, J. Chen, S.-J. Ge, Z.-H. Zhu, M.-J. Tang, R. Xu, W. Gao, T. Li, W. Hu, and Y.-Q. Lu, Adv. Mater. 30, 1705865 (2018).

[25] See Supplemental Material at http://link.aps.org/ supplemental/10.1103/PhysRevLett.120.213903 for another option to add a second space-variant cholesteric slab with opposite chirality and identical surface patterning, which is, however, more difficult to realize experimentally than a simple reflective coating at the back of the optical element. The working principle of such bilayered BraggBerry option is illustrated.

[26] L. Marrucci, E. Karimi, S. Slussarenko, B. Piccirillo, E. Santamato, E. Nagali, and F. Sciarrino, J. Opt. 13, 064001 (2011).

[27] D. W. Berreman, J. Opt. Soc. Am. 62, 502 (1972).

[28] We adopt the following convention for the complex representation of a paraxial beam propagating along the $\pm z$ axis. Namely, its complex amplitude is taken proportional to the propagation factor $\exp \left(-i \omega t \pm i k_{0} z\right)$ with $\omega$ the angular frequency, $t$ the time, and $k_{0}$ the free-space wave vector.

[29] V. A. Belyakov, Diffraction Optics of Complex-Structured Periodic Media (Springer, New York, 1992).

[30] P. Oswald and P. Pieransky, Nematic and Cholesteric Liquid Crystals: Concepts and Physical Properties Illustrated by Experiments (Taylor \& Francis, CRC, Boca Raton, FL, 2005).

[31] S. Erten, S. E. Swiontek, C. M. Graham, and A. Lakhtakia, J. Nanophoton. 9, 090599 (2015).

[32] L. Marrucci, C. Manzo, and D. Paparo, Phys. Rev. Lett. 96, 163905 (2006).

[33] G. Milione, H. I. Sztul, D. A. Nolan, and R. R. Alfano, Phys. Rev. Lett. 107, 053601 (2011).

[34] X. Yi, Y. Liu, X. Ling, X. Zhou, Y. Ke, H. Luo, S. Wen, and D. Fan, Phys. Rev. A 91, 023801 (2015).

[35] A. M. Beckley, T. G. Brown, and M. A. Alonso, Opt. Express 18, 10777 (2010). 